\title{
An Unusual Case of Pannus Formation - A Late Postoperative Complication of a Prosthetic Valve Replacement Causing Severe Cardiomyopathy
}

\author{
Carreon $\mathbf{A M}^{2} *$, Mohsin $\mathbf{I}^{1}$, Watson B', Carter RN ${ }^{2}$, \\ Ponder $\mathrm{M}^{3}$ and Kramer $\mathrm{K}^{3}$ \\ ${ }^{1}$ Department of Internal Medicine, Norton Community \\ Hospital- Internal Medicine Residency, Norton, United \\ States \\ ${ }^{2}$ Department of Cardiothoracic Surgery, Ballad CVA Heart \\ Institute, Kingsport, United States \\ ${ }^{3}$ Department of Cardiology, Ballad CVA Heart Institute, \\ Kingsport, United States \\ *Correspondling author: Carreon AM, Department of \\ Internal Medicine, Norton Community Hospital- Internal \\ Medicine Residency, Norton, United States
}

Received: March 15, 2021; Accepted: April 02, 2021; Published: April 09, 2021

\begin{abstract}
There are several well-known long-term complications following prosthetic valve replacement. Pannus formation is one of the rare complications whose estimated incidence varies between $0.3 \%$ and $1.3 \%$ per patient-year [1]. The exact etiology of pannus formation is unknown but histopathologically, pannus formation is due to fibroelastic hyperplasia that variably occurs after valve implantation [2]. The rarity and acute coronary syndrome like clinical presentation makes pannus formation a clinically important diagnosis during initial presentation. Workup preferably includes Transthoracic Echocardiography (TEE) with or without CT angiography. Management includes urgent or emergent surgical excision of the pannus with or without re-replacement of the aortic valve [1].

We present a 66-year-old female who presented with typical angina symptoms along with diffuse ischemic EKG changes five years after aortic valve replacement surgery with Trifecta Bioprosthesis due to severe aortic stenosis with Non-ST Elevation Myocardial Infarction (NSTEMI). Coronary angiography done on admission showed left main ostial stenosis of $90-95 \%$ in proximity to the aortic valve prosthesis. A Transthoracic Echocardiogram (TTE) revealed an ejection fraction of 30-35\% with global hypokinesia but did not suggest any valve dysfunction. CT angiogram was done the next day which revealed a noncalcified plaque (pannus) greater than $75 \%$ occlusion in the left main in proximity of the aortic valve. Despite expedited care and surgical evaluation, unfortunately the patient decompensated in the surgical operating room and did not survive.
\end{abstract}

Keywords: Pannus formation, Prosthetic aortic valve

\section{Introduction}

For patients who have severe aortic valve dysfunction, aortic valve replacement is recommended for preservation of left ventricular function, relief of symptoms, and patient survival [3]. The choice of prosthetic heart valve type should be a shared decision-making process. A mechanical valve has an advantage of being durable and long lasting while a bioprosthetic valve undergoes Structural Valve Deterioration (SVD) after six to eight years after implantation. However, mechanical valves are highly thrombogenic and require life-long anticoagulation therapy. Hence, bioprosthetic valves are preferred in patients with high risk of bleeding [4,5]. Age is important because incidence of SVD in a bioprosthetic valve is greater in young patients, while the risk of bleeding from prescribed anticoagulation post mechanical valve implantation is higher in older patients. Hence american heart association recommends bioprosthetic valves in patients of any age whom anticoagulants are contraindicated (class Ia). It is reasonable to implant an aortic mechanical valve for patients of age less than 50 years who can tolerate long term anticoagulation (class II a) and a bioprosthetic in aortic valve replacement is reasonable for patients of age more than 70 years (class II a) $[5,6]$.

Occurrences of 30-day mortality after aortic valve replacement have been documented at approximately $2.5 \%$. Early complications include: atrial fibrillation, high grade AV block with pacemaker implantation, renal failure requiring dialysis, stroke, and endocarditis [7]. Pannus formation is an uncommon but serious complication of prosthetic valve replacement. It is one of the two causes of structural acquired Prosthetic Valve Obstruction (PVO), the other being thrombus formation. It is likely due to fibroelastic hyperplasia which occurs over time and usually restricts normal leaflet motion [8]. There are currently no guidelines for preventing pannus formation since it

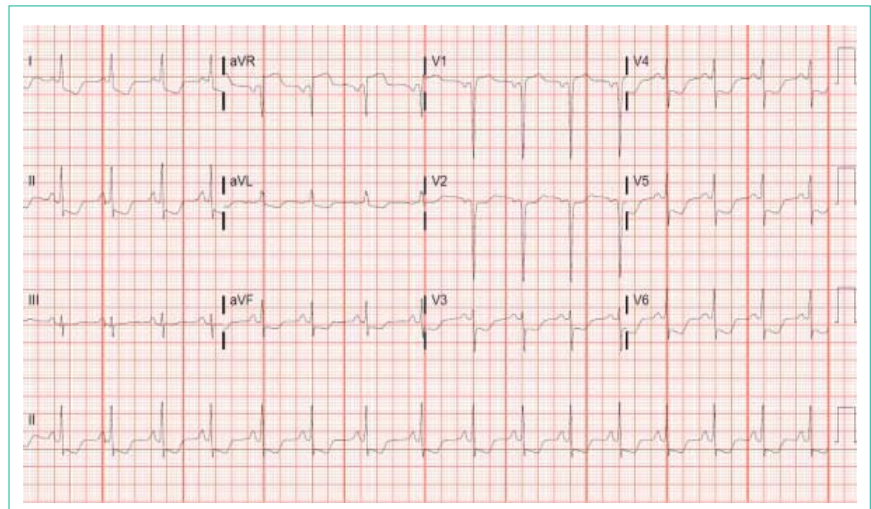

Figure 1: EKG with subtle ST elevation in AVR, V1 and diffuse ST segment depression in multiple leads.
Austin J Clin Cardiolog - Volume 7 Issue 1 - 2021

ISSN : 2381-9111 | www.austinpublishing group.com

Carreon et al. (C) All rights are reserved
Citation: Carreon AM, Mohsin I, Watson B, Carter RN, Ponder M and Kramer K. An Unusual Case of Pannus Formation - A Late Postoperative Complication of a Prosthetic Valve Replacement Causing Severe Cardiomyopathy. Austin J Clin Cardiolog. 2021; 7(1): 1076 
is usually a complication of chronic underlying processes [9]. The incidence varies from $0.73 \%$ in bi-leaflet valves to $1.8 \%$ in tilting disc valves and occurs in both mechanical and bioprosthetic valves $[1,10]$. The timeline for occurrence ranges from days to over 5 years, with an average of $24 \pm 26$ months. Echocardiography is the main diagnostic test to evaluate prosthetic valve function and regular follow up is standard of care [1].

\section{Case Presentation}

In 2015, a 66-year old female underwent a $19 \mathrm{~mm}$ Trifecta bioprosthetic Aortic Valve Replacement (AVR) secondary to severe calcified aortic stenosis. Significant medical history includes hypertension, hyperlipidemia, and hypothyroidism. She presented to the emergency department at an outside facility with complaints of intermittent chest pain consistent with typical angina that was graded severe, pressure type, mid sternal in location, and radiated throughout the chest wall. She acknowledged a history of smoking, illicit drug abuse, and chronic narcotic dependence. The patient had poor compliance and no follow-up after AVR. Physical examination was unremarkable, S1-S2 normal. EKG revealed sinus rhythm with subtle ST segment elevation in AVR, V1 and diffuse ST segment depression in multiple leads concerning left main/multivessel CAD (Figure 1). Troponin levels were 1.88 with a repeat value of 2.37 on serial measurements. The patient was given ticagrelor, aspirin, heparin, metoprolol, and nitroglycerine paste, and referred to our hospital for expedited cardiac evaluation.

The patient underwent emergent Left Heart Catheterization (LHC), which showed ostial left main 90-95 \% stenosis in proximity to aortic valve bioprosthesis, which implied a mechanical obstruction (Figure 2). Left anterior descending artery, left circumflex artery (dominant), and posterior descending artery from the left system was normal. Left ventricle was not crossed because of the presence of the prosthetic aortic valve and was evaluated with a $2 \mathrm{D}$ echo after the LHC. Ticagrelor was stopped and CT surgery was consulted for a possible coronary bypass surgery grafting.

2D Echocardiography revealed an ejection fraction of 30-35\%, global hypokinesia, and a well-seated bioprosthetic aortic valve with peak gradient and mean gradient of $25 \mathrm{mmHg}$ and $14 \mathrm{~mm} \mathrm{Hg}$ respectively, which is considered normal for this bioprosthetic aortic valve (Figure 3). CT Angiogram was done the next day and showed a non-calcified plaque (pannus) greater than $75 \%$ occlusion in the left

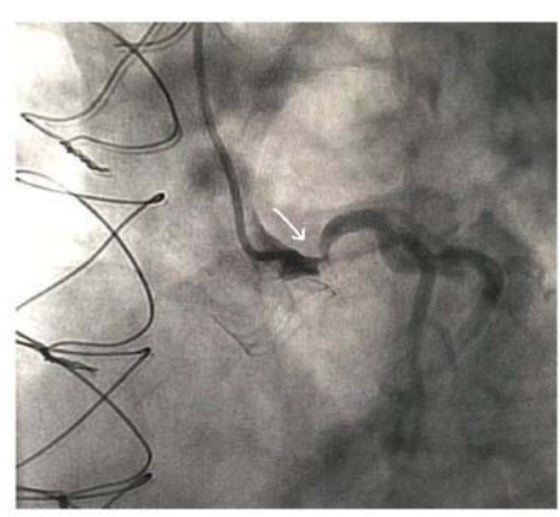

Figure 2: Left heart catheterization showing ostial left main $90-95 \%$ stenosis.

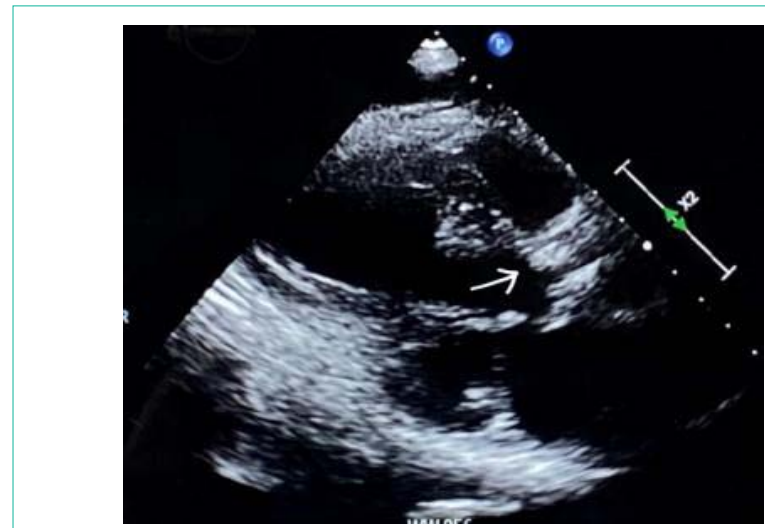

Figure 3: 2D Echo showing a well-seated bioprosthetic aortic valve.

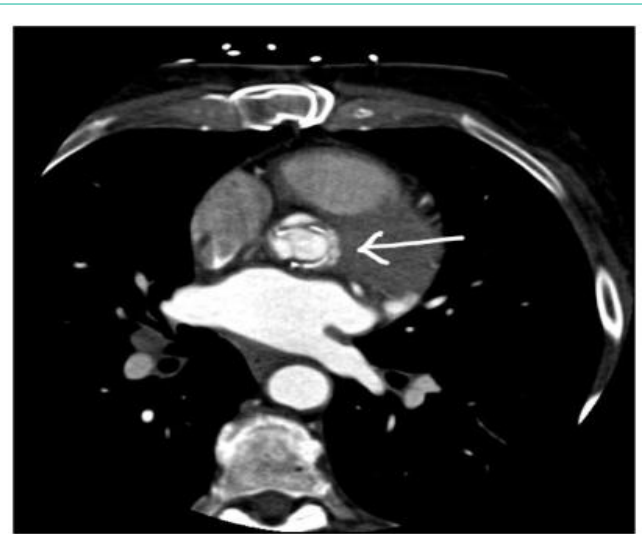

Figure 4: CT Angiogram showing a non-calcified plaque greater than $75 \%$ occlusion in the proximity of the aortic valve.

main in proximity of the aortic valve (Figure 4). The patient was taken to the operation room for a redo sternotomy and pannus excision. Unfortunately, the patient went into ventricular fibrillation cardiac arrest in the operating room, after induction of anesthesia. Despite multiple resuscitative efforts as per ACLS protocol, the patient did not survive.

\section{Discussion/Conclusion}

The Trifecta bioprosthetic valve is made by St. Jude Medical and was approved for use by the FDA in 2011. Its design includes pericardial tissue leaflets attached to the exterior of the valve to improve the opening area, along with a titanium stent. It is intended to reduce the risk of abrasion and valve deterioration during the cardiac cycle. Due to its relatively recent release, late complications for this specific type of valve replacement are not well known. Early documented studies have shown that this valve has promising results with good hemodynamic performance. Due to its design, undersizing or oversizing must be avoided to reduce unnecessary implantation difficulty and cardiac gradient increase due to excess prosthetic leaf tissue [7].

Early bioprosthetic valve failure usually occurs within five years of the index surgery. Known risk factors for accelerated calcification include younger age, enhanced immune response, hyperparathyroidism, diabetes mellitus, and mitral (as opposed to 
aortic) position [11]. Late complications include: valve obstruction, embolic events, bleeding, prosthetic valve dysfunction, prosthesis mismatch, and infective endocarditis.

Thrombosis and pannus formation are known mechanical causes of valve obstruction.

Echocardiography is the diagnostic study of choice after valve replacement and should be performed at discharge or in thirty days and six to twelve months after the procedure or when valve dysfunction is suspected. It should also be noted that regular follow up is recommended after five years for patients with bioprosthetic valves. Decisive treatment of choice in critically ill patients usually involves surgery. Overall mortality ranges from $4-5 \%$ in patients presenting with NYHA Class III failure and goes up to as high as $15-20 \%$ in patients presenting with NYHA Class IV failure [1]. Pannus formation occurs less commonly than thrombosis. One study estimates that $77 \%$ of valve obstruction is due to thrombosis as opposed to $11 \%$ that is due to pannus formation [12].

Pannus formation is thought to be due to fibroelastic hyperplasia, which occurs over time [2]. The effect of pannus formation on hemodynamics depends on the extent of narrowing of the outflow tract and its effect on the motion of the prosthetic leaflets. Severe stenosis may occur from a large circumscribed pannus. In the worst cases, it can present as cardiogenic shock in patients with a history of valve replacement. This effect of pannus formation is an underrecognized cause of valve obstruction [10].

Pannus formation is a rare complication of valve replacement. Most documented cases of pannus formation are associated with mechanical valve replacements. To our knowledge, this is the first documented pannus formation that is formed just adjacent encroaching on LMCA. Complicating factors that occurred during hospitalization were: development of cardiogenic shock and patient noncompliance with continued drug use, as well as disregard for guidelines on follow up. This case illustrates the importance of regular long-term follow-up after AVR. Late complication like pannus formation is uncommon but yield serious complications like cardiogenic shock and death. Hence, recognition of this complication in patients during late postoperative periods is critical. Our case report can contribute to the late complications of prosthetic valve replacement, which needs further study.

\section{References}

1. Philippe Pibarot, Jean G. Dumesnil. Valvular heart disease: changing concepts in disease management. Circulation. 2009; 119: 1034-1048.

2. Ellensen VS, Andersen KS, Vitale N, Davidsen ES, Segadal L, Haaverstad R. Acute Obstruction by Pannus in Patients with Aortic Medtronic-Hall Valves: 30 years of Experience. Annals of Thoracic Surgery. 2013; 96: 2123-2128.

3. Chiang YP, Chikwe J, Moskowitz AJ, Itagaki S, Adams DH, Egorova NN Survival and Long-term Outcomes Following Bioprosthetic vs Mechanical Aortic Valve Replacement in Patients Aged 50 to 69 Years. JAMA. 2014; 312: 1323-1329.

4. Glauber M, Lio A, Miceli A. Sutureless technology for aortic valve replacement: Looking beyond crossclamptime. J Thorac Cardiovasc Surg. 2016; 151: 1637-1638.

5. Nishimura RA, Otto CM, Bonow RO, Carabello BA, Erwin JP, Fleisher LA, et al. A2017 AHA/ACC Focused update of the 2014 AHA/ACC guideline for the management of patients with valvular heart disease: A report of the American College of Cardiology/ American Heart Association task force on clinical practice guidelines. Circulation. 2017; 135: 1159-1195.

6. Glaser N, Jackson V, Holzmann MJ, Franco-Cereceda A, Sartipy U. Aortic valve replacement with mechanical vs. biological prostheses in patients aged 50-69 years. Eur Heart J. 2016; 37: 2658-2667.

7. Permanyer E, Estigarribia AJ, Ysasi A, Herrero E, Semper O, Llorens R, St. Jude Medical Trifecta $^{\mathrm{TM}}$ aortic valve perioperative performance in 200 patients, Interactive CardioVascular and Thoracic Surgery. 2013; 17: 669672.

8. Moss AJ, Dweck MR, Dreisbach JG, Williams MC, Mak SM, Cartidge T, et al. Complementary role of cardiac CT in the assessment of aortic valve replacement dysfunctionOpen Heart. 2016; 3: e000494.

9. Bonnichsen CR, Pellikka PA. Prosthetic Valve Thrombus Versus Pannus. Circulation: Cardiovascular Imaging. 2015; 8.

10. Darwazah AK. Recurrent pannus formation causing prosthetic aortic valve dysfunction: Is excision without valve re-replacement applicable?. J Cardiothorac Surg. 2012; 7: 62.

11. Cremer PC, Rodriguez LL, Griffin BP, Tan C, Rodriguez R, Johnston DR, et al. Early Bioprosthetic Valve Failure: A Pictorial Review of Rare Causes,JACC: Cardiovascular Imaging. 2015; 8: 737-740.

12. Pislaru S, Connolly HM. Clinical manifestations and initial evaluation of prosthetic valve thrombosis and obstruction. 2020. 